\title{
Rethinking the Roles of Pathogens and Mutualists: Exploring the Continuum of Symbiosis in the Context of Microbial Ecology and Evolution
}

\author{
Ashley Stengel, ${ }^{1,2,3, \dagger}$ Rhae A. Drijber, ${ }^{2}$ Erin Carr, ${ }^{4}$ Thais Egreja, ${ }^{3}$ Edward Hillman, ${ }^{5}$ Timothy Krause, ${ }^{4}$ Samantha Reese, ${ }^{4}$ and \\ Joshua R. Herr ${ }^{3,4,6, \dagger}$ \\ ${ }^{1}$ Complex Biosystems Interdisciplinary Life Sciences Program, University of Nebraska, Lincoln, NE 68588 \\ ${ }^{2}$ Department of Agronomy and Horticulture, University of Nebraska, Lincoln, NE 68583 \\ ${ }^{3}$ Department of Plant Pathology, University of Nebraska, Lincoln, NE 68583 \\ ${ }^{4}$ Genetics, Cell, and Molecular Biology Section, School of Biological Sciences, University of Nebraska, Lincoln, NE 68583 \\ ${ }^{5}$ Department of Plant Pathology and Genetics, Cell, and Molecular Biology Section, School of Biological Sciences, University of Nebraska, \\ Lincoln, NE 68583 (deceased) \\ ${ }^{6}$ Center for Plant Science Innovation, University of Nebraska, Lincoln, NE 68588
}

Accepted for publication 10 January 2022.

\begin{abstract}
Systems of classification are important for guiding research activities and providing a common platform for discussion and investigation. One such system is assigning microbial taxa to the roles of mutualists and pathogens. However, there are often challenges and even inconsistencies in reports of research findings when microbial taxa display behaviors outside of these two static conditions (e.g., commensal). Over the last two decades, there has been some effort to highlight a continuum of symbiosis, wherein certain microbial taxa may exhibit mutualistic or pathogenic traits depending on environmental contexts, life stages, and plant host associations. However, gaps remain in understanding how to apply the continuum approach to host-microbe pairs across a range of environmental and ecological factors. This commentary presents an alternative framework for evaluating the continuum of symbiosis using dominant archetypes that define symbiotic ranges. We focus
\end{abstract}

\section{ABSTRACT}

particularly on fungi and bacteria, though we recognize that archaea and other microeukaryotes play important roles in host-microbe interactions that may be described by this approach. This framework is centered in eco-evolutionary theory and aims to enhance communication among researchers, as well as prioritize holistic consideration of the factors shaping microbial life strategies. We discuss the influence of plant-mediated factors, habitat constraints, coevolutionary forces, and the genetic contributions which shape different microbial lifestyles. Looking to the future, using a continuum-of-symbiosis paradigm will enable greater flexibility in defining the roles of target microbes and facilitate a more holistic view of the complex and dynamic relationship between microbes and plants.

Keywords: agriculture, ecology, genomics, microbiome, plant pathology, symbiosis
Corresponding authors: A. Stengel; ashsten@gmail.com, and J. R. Herr; jherr@unl.edu

Funding: Although funding sources did not directly fund the writing of this document, some of the authors here have been supported by federal research funds. J. R. Herr acknowledges support from United States National Science Foundation (US-NSF) Established Program to Stimulate Competitive Research (EPSCoR) grant 1557417, US-NSF grant 1560163, National Air \& Space Administration Grant 80NSSC17K0737, and National Institute of Justice grant 2017-IJ-CX-0025, all of which contributed to the support of some of these authors.

The author(s) declare no conflict of interest.

Copyright (C) 2022 The Author(s). This is an open access article distributed under the CC BY 4.0 International license.
It is a human trait-one that is particularly pronounced among biologists - to want to create order out of chaos. To understand the types of interactions that may occur between organisms in the natural world, researchers have developed categories defined by the life history traits observed for a particular organism. Discrete naming conventions regarding host-microbe associations are based on host outcomes, such that harmful interactions are labeled pathogenic while beneficial interactions are labeled mutualistic (Fig. 1A). In research, teaching, and science communication, these static categories are used in conjunction with the disease triangle to offer a conceptual paradigm for evaluating complex interactions between hosts, microbes, and the environment (Box 1). Although the scientific literature recognizes fluidity in host-microbe interactions (Johnson et al. 1997; Martino et al. 
2018; Smith et al. 2017), many of these concepts are often simplified into mutually exclusive categories. Simplifying these complex and dynamic relationships is considered advantageous for streamlining analysis of research findings and teaching material; however, this approach can impede holistic understanding of real-world interactions (Newton et al. 2010). Furthermore, absence of a well-defined conceptual approach for studying variable microbial life histories has led to significant knowledge gaps in studies of disease pathology (Casadevall and Pirofski 2003), microbial ecology (Saikkonen et al. 2020), and the role of abiotic and biotic factors on symbiosis outcomes (Johnson et al. 2010; Rudgers et al. 2020). Given that microbial associations vary among plant hosts (Lofgren et al. 2018;

A

Static Host-Microbe Associations

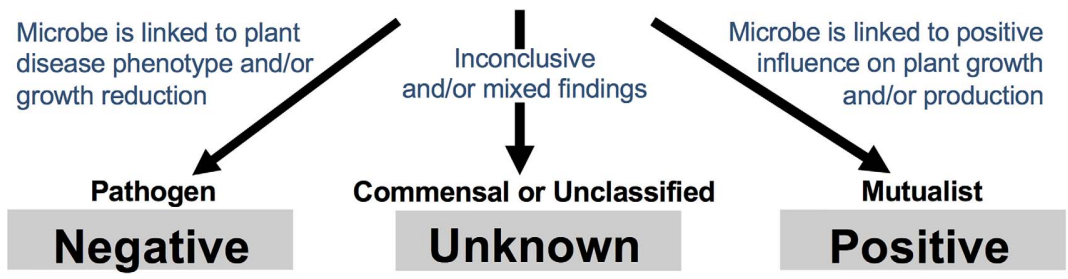

B Dynamic Host-Microbe Associations

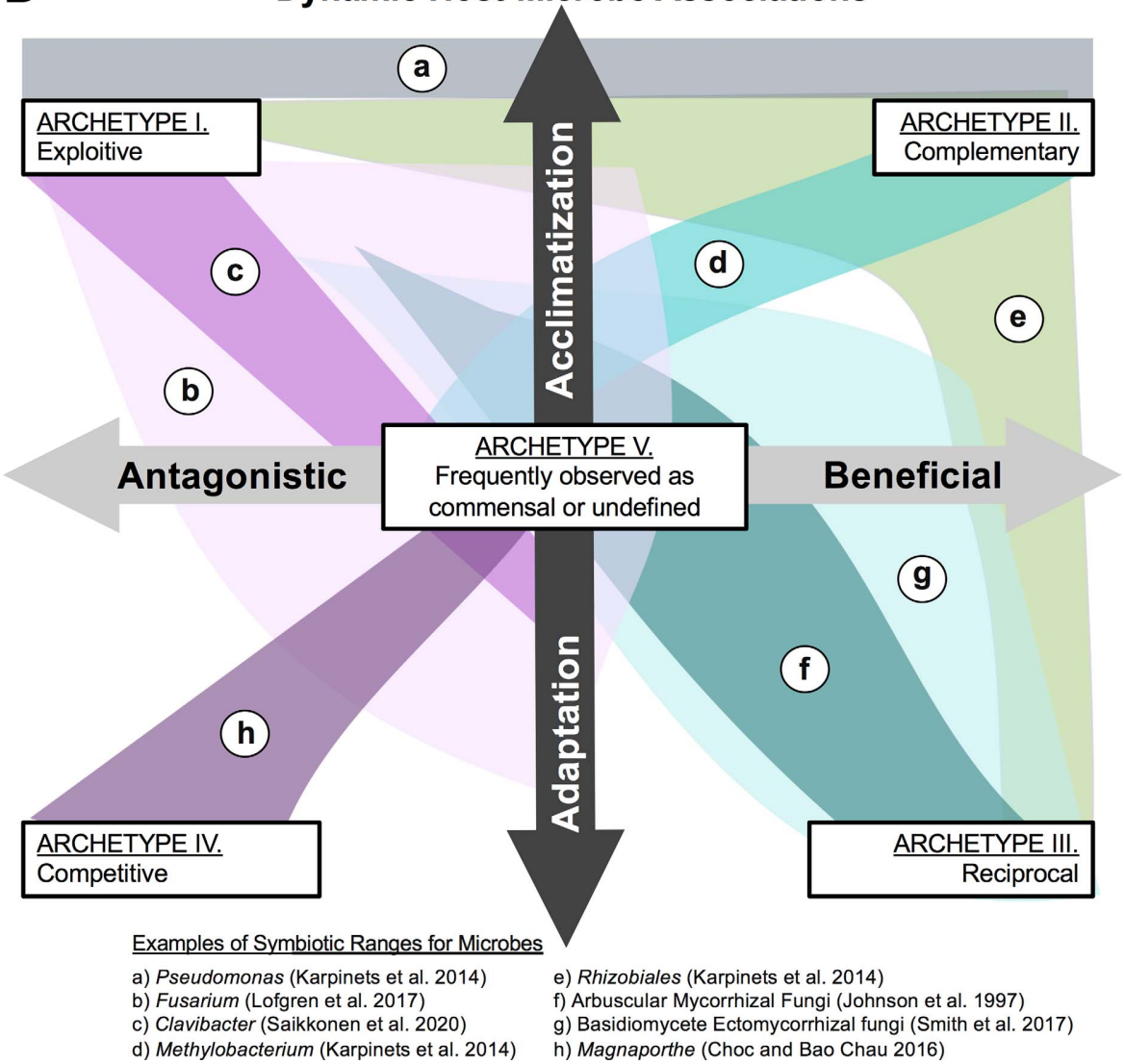

Fig. 1. Two competing conceptual frameworks for evaluating, classifying, and studying plant-microbe symbiosis. A, Static, traditional approach to assigning life history roles to pathogens, mutualists, and those with an unknown role (commensals). B, The continuum approach considers symbiotic ranges that fit across dominant "archetypes" of host-microbe associations and uses an eco-evolutionary framework to account for plasticity and adaptability in host-microbe associations over time scales. The horizontal arrow in light gray represents the range of phenotypes in host-microbe interactions, ranging from antagonistic or negative (left) to beneficial or positive (right). The vertical arrow in dark gray represents the spatiotemporal variability of host-microbe interactions, including associations or functional roles that are transient or highly responsive to environmental and ecological factors (acclimatization: top) and those that are stable or exhibit lower variation over a range of environmental, host, and ecological factors (adaptation: bottom). Using this scale, symbiotic ranges fit across five dominant archetypes. The top left quadrant represents dynamics that are highly context dependent and contribute to negative interactions, such as a host being parasitized by a microbe (archetype I, exploitive acclimatization). The top right quadrant represents highly transient interactions that facilitate mutual benefits to host and microbial organisms under certain environmental or ecological conditions (archetype II, complementary acclimatization). The bottom right quadrant represents evolutionarily stabilized plant-microbe interactions wherein both organisms are benefitted through the association (archetype III, reciprocal adaptation). The bottom left quadrant represents tightly linked competitive relationships between plants and microbes that contribute to coevolutionary arms races, such as microbial pathogenesis and host defense mechanisms (archetype IV, competitive adaptation). The center of the diagram represents commensal or unknown relationships between plants and microbes, wherein phenotypic outcomes are neither positive or negative, and there are myriad gene by gene by environment $(G \times G \times E)$ interactions. Boxes with letters (a to $h)$ correspond to examples of symbiotic ranges, with microbial organisms listed in the legend. 


\section{BOX 1}

\section{Continuum of symbiosis paradigm avoids oversimplification and integrates multipartite complexity into the study of plant-microbe interactions}

The disease triangle is introduced as a foundational paradigm in the study of plant pathology (Figure I). Educators present the triangle as a key component of course curricula, researchers may use the triangle to contextualize their experiments, and extension and outreach professionals use the triangle to communicate with stakeholders about disease outbreaks. While this paradigm aims to simplify complex dynamics that characterize host-microbe-environment interactions, the conceptualization fails to account for beneficial or commensal relationships and ignores the plasticity in host and microbe expression that can give rise to a spectrum of phenotypes. As such, symbiosis outcomes are over-simplified giving rise to 'one-host, onepathogen' characterizations (Figure II). Consequently,

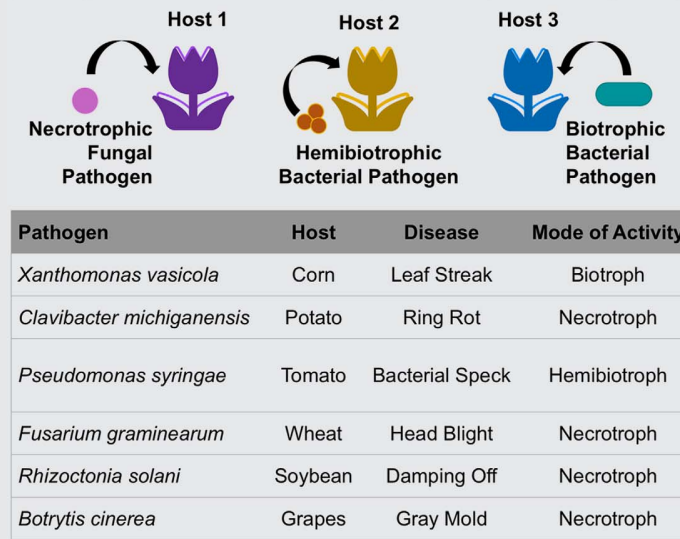

Figure II. Singular Host-Pathogen Interactions

Examples of pathogenesis are primarily characterized through presence/absence of host-microbe pairs. Some notable examples include necrotrophic fungi and bacteria displaying a range of pathogenic trophic modes. The assumption is that when this microbe is present, disease will occur, regardless of additional context from plant-microbe, microbemicrobe, and/or microbe-environment interactions.

with characteristic phenotypes and additional (Figure III). Conceptually, it contextualizes plant-microbe interactions within the ecological dynamics of community-level processes, such as competition or cooperation, and environmental features, such as nutrient availability or habitat constraints. This provides researchers an improved framework for evaluating host-microbe interactions amidst several selection pressures, including cases of plasticity and reciprocal selection that facilitate short-term acclimatization and stabilized patterns of co-adaptation. The continuum paradigm also offers a shift in teaching pedagogy that corrects common misconceptions about host-microbe interactions, thereby facilitating more holistic and comprehensive interpretations of symbiosis outcomes. Incorporating the continuum of symbiosis paradigm harbors great potential for improving research initiatives, scientific communication, and student learning gains across disciplines studying host-microbe interactions.

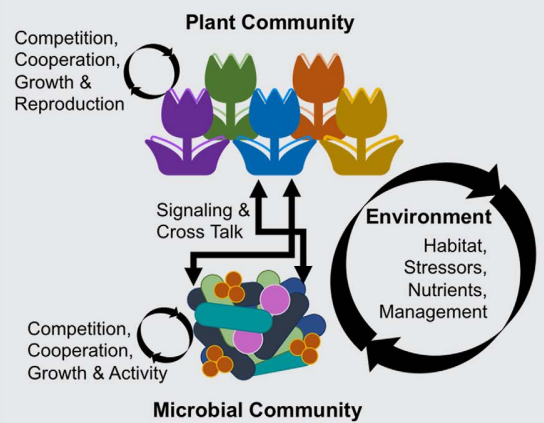

Figure III. Continuum of Symbiosis

Community-level processes, spatiotemporal dynamics, environmental factors, evolutionary relationships, and management practices all interact to describe dis outcomes. Actapting our plant-microbe interaction paraoutcomes. Adapting our plant-microbe interaction para-
digms to fully consider these factors will improve research, teaching, and communication outcomes across plant pathology and related disciplines.

\begin{tabular}{|c|c|c|c|}
\hline Host-Microbe Pair & Environment & Symbiosis Outcomes & Archetype Features \\
\hline $\begin{array}{l}\text { Vaccinium myrtillus \& } \\
\text { Oidiodendron maius } \\
\text { (Martino et al. 2017) }\end{array}$ & $\begin{array}{l}\text { Greenhouse } \\
\text { experiment } \\
\text { using fungal } \\
\text { isolates }\end{array}$ & $\begin{array}{l}\text { Mutualism: increases } \\
\text { plant biomass } \\
\text { Commensalism: no } \\
\text { change in plant host }\end{array}$ & $\begin{array}{l}\text { Archetype III. Ericoid } \\
\text { mycorrhizal fungi, } \\
\text { upregulation of CAZy } \\
\text { enzymes, lipases, and } \\
\text { proteases to support } \\
\text { nutrient acquisition and } \\
\text { exchange with plant host }\end{array}$ \\
\hline $\begin{array}{l}\text { Tobacco \& Glomus } \\
\text { macrocarpum } \\
\text { (Reviewed in Johnson et } \\
\text { al. 1997) }\end{array}$ & $\begin{array}{l}\text { Intensively } \\
\text { managed } \\
\text { agricultural } \\
\text { system in } \\
\text { Kentucky, USA } \\
\text { with extremely } \\
\text { high soil } \\
\text { phosphorous }\end{array}$ & $\begin{array}{l}\text { Antagonism: decreases } \\
\text { above-ground biomass, } \\
\text { root length and flowering } \\
\text { Commensalism: no } \\
\text { harmful effects observed } \\
\text { on other crop species in } \\
\text { the same field or when } \\
\text { tobacco is grown in } \\
\text { rotation with Festuca }\end{array}$ & $\begin{array}{l}\text { Archetypes I and } V \text {. } \\
\text { Arbuscular mycorrhizal } \\
\text { fungi, environmental } \\
\text { conditions and plant } \\
\text { species identity facilitate } \\
\text { transiently antagonistic } \\
\text { associations among a } \\
\text { microbial group known for } \\
\text { its tightly linked mutualism }\end{array}$ \\
\hline $\begin{array}{l}\text { Chorispora bungeana } \\
\text { \& Clavibacter } \\
\text { michiganensis - strain } \\
\text { Enf12 } \\
\text { (Reviewed in Saikkonen } \\
\text { et al. 2020) }\end{array}$ & $\begin{array}{l}\text { Subnival alpine } \\
\text { plants } \\
\text { maintained at } \\
\text { chilled }\left(0^{\circ} \mathrm{C}\right) \\
\text { temperatures }\end{array}$ & $\begin{array}{l}\text { Mutualism: increases } \\
\text { cold tolerance of host } \\
\text { plants }\end{array}$ & $\begin{array}{l}\text { Archetype II or III. } \\
\text { Endophytic bacteria, } \\
\text { increased activities of } \\
\text { antioxidant enzymes, and } \\
\text { proline content which } \\
\text { support host survival } \\
\text { under stress }\end{array}$ \\
\hline $\begin{array}{l}\text { Nicotiana } \\
\text { benthamiana } \\
\text { \& Rhodococus } \\
\text { isolates }\end{array}$ & $\begin{array}{c}\text { Greenhouse } \\
\text { experiment } \\
\text { using isolates } \\
\text { from a Pistachio } \\
\text { nursery }\end{array}$ & $\begin{array}{l}\text { Antagonism: leafy gall } \\
\text { symptoms } \\
\text { Mutualism: promotes } \\
\text { growth of root hairs and } \\
\text { lateral roots }\end{array}$ & $\begin{array}{l}\text { Archetypes I and II. } \\
\text { Bacteria, presence or } \\
\text { absence of virulence } \\
\text { gene triggers shift in } \\
\text { microbial life strategy }\end{array}$ \\
\hline
\end{tabular}


Saikkonen et al. 2016) and are fluid across spatiotemporal scales (Hawkes and Kiett 2015; Rudgers et al. 2020), there is need for a holistic framework for evaluating microbe-host interactions.

In this commentary, we build on the continuum-of-symbiosis approach and propose a new unifying framework that recognizes plasticity and adaptability in microbe-host-environment interactions across spatial and temporal scales. We highlight that lifestyles and strategies employed by microorganisms are not static in nature and, instead, are defined by adapting, shifting, and evolving to overcome constraints. Clearly defining symbiosis outcomes necessitates some focus on singular microbial taxa and plant host combinations (Plett and Martin 2011). However, within natural systems, these interactions occur in the broader context of mixed plant and microbe community dynamics (Kohl 2020; Rudgers et al. 2020; Toju et al. 2018). As such, the definitions and concepts used to describe host-microbe interactions should reflect these dynamic processes and support observations across a range of environmental and ecological contexts. Thus, this new conceptual framework integrates eco-evolutionary dynamics to consider how the ecology of organisms and their environment contribute to differing evolutionary trajectories that shape symbiosis outcomes. This paradigm transition will be important for advancing host-microbe research, and for improving scholarship by encouraging researchers, educators, and students to rethink how host-microbe interactions contribute to and are shaped by natural variability and diversity.

\section{HISTORY OF THE CONTINUUM OF SYMBIOSIS}

The continuum of symbiosis is not a new concept. Johnson et al. (1997) applied the continuum approach to mycorrhizae to more effectively describe diversity and variability in plant-microbe associations across a range of abiotic and biotic factors. The goal of the continuum approach is to modify how researchers conceptualize host-microbe interactions to recognize the role of transience, plasticity, and adaptability in host-microbe-environment interactions (Hawkes and Kiett 2015; Johnson et al. 1997; Newton et al. 2010; Rudgers et al. 2020). Using this approach, a microbe may only fulfill a mutualistic role under certain conditions or in connection with a specific host plant, instead of always and obligately being a mutualist (Casadevall and Pirofski 2003; Johnson et al. 2010; Martino et al. 2018; Plett and Martin 2011; Saikkonen et al. 2020). It is important to note that these relationships are not formed singularly or in isolation and, as such, the broader ecology of the system can shape host and microbe responses (Casadevall and Pirofski 2003; Hawkes and Kiett 2015; Saikkonen et al. 2020). These features make the continuum approach well suited for integrating ecoevolutionary dynamics to improve research conceptualizations and study designs for investigating host-microbe interactions (Box 1).

Eco-evolutionary dynamics represent a conceptual framework that acknowledges that ecological process and evolutionary dynamics act on each other. In other words, ecological and evolutionary processes shape one another through reciprocal and interacting influences (Brunner et al. 2019). In the context of host-microbe interactions, this includes processes such as microbiome assembly, diversity, and function, as well as a myriad of abiotic and biotic factors that further influence microbe or host fitness and evolution (McDonald et al. 2020; Saikkonen et al. 2020; Trivedi et al. 2020). As such, applying an eco-evolutionary framework to the study of plant-microbe interactions may include evaluating how symbiotic relationships change across habitat conditions (Hawkes and Kiett 2015; Martino et al. 2018), among plant hosts (Lofgren et al. 2018; Saikkonen et al. 2016), in response to environmental perturbations (Jones et al. 2019), along climate gradients (Rudgers et al. 2020), or over different time scales (Morella et al. 2020). Enabling flexibility in how we describe complex interactions will support future research efforts as we prioritize a more holistic examination of the factors shaping plant-microbe interactions. This transition is also relevant to our teaching practices. Traditional textbook examples of pathogen-host interactions typically include highly specific "one host-one interaction" relationships (Box 1). Although these cases may provide an overview of distinctions that are useful as teaching examples, they fall short in providing an evidence-based foundation for applying examples to research and practical management scenarios.

Here, we propose that the spectrum of behaviors demonstrated by microbes may be evaluated as symbiotic ranges fitting within dominant "archetypes" ranging from antagonistic to beneficial interaction phenotypes, and from transient and highly context dependent (acclimatization) to stabilized and less spatially or temporally variable (adaptation) (Fig. 1B). Acclimatization is a short-term strategy enabling organisms to adapt to naturally occurring changes in the environment, thus giving rise to interaction phenotypes that are more spatiotemporally variable (Hawkes and Kiett 2015). In contrast, adaptation is an evolutionary process where natural selection gives rise to genetic changes that improve organismal fitness under certain environmental conditions (Hawkes and Kiett 2015). As such, adaptation is indicative of interaction phenotypes that are less spatiotemporally variable and more stable. Using these two scales, one for spatiotemporal variability (Fig. 1B, vertical arrow) and one for interaction phenotypes (Fig. 1B, horizontal arrow), we can better contextualize how ecological and evolutionary processes contribute to plasticity in symbiosis outcomes. We present five archetypes that describe symbiosis outcomes across these scales:

- Archetype I. Exploitive acclimatization:

- Antagonistic interactions between microbes and hosts

- Temporally or spatially variable across environmental conditions or ecological pressures

- Short-lived associations that preferentially support survival of either microbe OR host under eco-environmental constraints

- Symbiosis outcomes are highly responsive to changes/ shifts in surroundings

- Archetype II. Complementary acclimatization:

- Beneficial interactions between microbes and hosts

- Temporally or spatially variable across environmental conditions or ecological pressures

- Short-lived associations that promote microbe AND host survival under eco-environmental constraints

- Symbiosis outcomes are highly responsive to changes/ shifts in surroundings

- Archetype III. Reciprocal adaptation:

- Beneficial interactions between microbes and hosts

- Stable across a range of environmental conditions or ecological pressures, including geographic location, host species identity, or climate regimes

- Longer-lived associations promoting microbe AND host survival through reciprocal selection and coadaptation (mutualisms)

- Symbiosis outcomes are less variable (resistant or resilient) in response to short-term changes/shifts in surroundings

- Archetype IV. Competitive adaptation: Antagonistic interactions between microbes and hosts Stable across a range of environmental conditions or ecological pressures giving rise to predictable patterns of host-microbe associations 
- Longer-lived associations promoting microbe OR host survival through competitive coadaptations and co-selection

Symbiosis outcomes are less variable (resistant or resilient) in response to short-term changes/shifts in surroundings

- $\quad$ Archetype V. Commensal:

- Recognized coexistence with neither distinctively antagonistic or beneficial associations

- Unknown or uncharacterized variability across environmental and ecological pressures that do not indicate types or strength of fitness outcomes for host or microbes

- Symbiosis outcomes demonstrate unclear or uncharacterized responses to short or long-term changes/shifts in surrounding

We suggest that evaluating symbiotic ranges across dominant archetypes offers an improved research and education framework that complements research findings and supports subsequent application of dynamic host-microbe associations to realworld scenarios (Box 1). For example, ectomycorrhizal (ECM) fungi can transition between saprophytic and biotrophic lifestyles (Fig. 1B-g), demonstrating transitory antagonism (archetype I) that can arise from a commensal lifestyle (archetype V), as well as beneficial associations that encompass short-lived exchanges (archetype II) and coevolved mutualisms with a plant host (archetype III). Similarly, the continuum approach recognizes that Pseudomonas spp. (Fig. 1B-a) can be found as highly opportunistic plant antagonists (archetype I) as well as plant-growthpromoting organisms that benefit certain plant hosts under a subset of environmental conditions (archetype II). This perspective addresses the roles of plant hosts, environmental conditions, spatiotemporal dynamics, and microbial genomes in shaping symbiotic relationships. We conclude by identifying ways forward that build on the continuum of symbiosis as a conceptual alternative to enhance understanding of plant-microbe interactions.

\section{PLANT-MEDIATED FACTORS CONTRIBUTE TO PATTERNS OF MICROBIAL SYMBIOSIS}

Plant host factors play an important role in shaping the trajectory of microbial interactions. Host species identity, host age or growth stage, and plant immune response are just a few of the factors that have been shown to alter symbiosis outcomes. Host species identity has a pronounced impact on the structure and function of the host-associated microbiome: directly by filtering and selecting microbes from the community (Kohl 2020) and indirectly through modification of the environment (Jones et al. 2019). Thus, the role assumed by a microbial group in association with a plant host is influenced by host-specific factors and reflects dynamic selection pressures that may give rise to coadapted relationships between host and microbe. For example, Fusarium isolates derived from nonsymptomatic North American native grasses were fully capable of causing head blight on wheat (Lofgren et al. 2018). This variability in symbiosis outcomes across plant hosts suggests that the genus Fusarium has a wide symbiotic range (Fig. 1B-b, represented by the light pink color) characterized by highly transient associations between hosts and microbes ranging from antagonistic (archetype I) to commensal (archetype V), as well as some stable, coadapted relationships (archetypes III and IV). In opposition to this highly flexible symbiotic range, Clavibacter spp. (Fig. 1B-c, represented by the dark pink color) exhibit a much smaller range of interaction between commensal (archetype V) and extremely antagonistic (archetype I) phenotypes. In these examples, plant hosts are exerting selection pressures on microbial communities that influence microbial fitness and shape outcomes of symbiosis (Fig. 2A and B).
These pressures are driven, in part, by host-specific features (e.g., species identity) and temporal shifts corresponding to host age.

Both the species identity and growth stage of a plant influence the production and release of phytohormones (Chaparo et al. 2014; Trivedi et al. 2020). These plant-mediated signals have diverse effects on the resident microbial community, in some cases contributing to the inhibition of microbial growth (Fig. 2A) and, in other cases, facilitating recruitment and modification of the rhizosphere to attract microbes (Fig. 2B). During early growth stages, Arabidopsis roots release carbon-rich substrates facilitating microbial colonization dominated by Actinobacteria and Acidobacteria (Chaparro et al. 2014). However, at later growth stages, root compounds are predominately antimicrobial, which coincides with the enrichment of Bacteroidetes and Cyanobacteria, highlighting the role of plant signaling in shaping microbial community composition (Chaparro et al. 2014). Host signals and subsequent cross-talk between plant phytohormones and microbial communities enable some symbiotic ranges to expand while reducing the range of others. For example, Arabidopsis mutants with disrupted jasmonic acid pathways give rise to different patterns of root exudation that change the overall bacterial community structure and may facilitate host resistance to particular groups of fungi (Jones et al. 2019).

Damage experienced by plant hosts is another important factor shaping symbiosis outcomes. In particular, defense phytohormones associated with systemic acquired resistance and induced systemic resistance pathways can shape microbial community composition (Jones et al. 2019; Trivedi et al. 2020). Furthermore, as phytohormone signaling changes in response to damage, it can indirectly shape trophic relationships. In this way, induced resistance can trigger host defense against a broad range of microbes, including endophyte, epiphyte, and rhizosphere communities (Newton et al. 2010). The damage response framework explains that microbial pathogenesis is influenced by a combination of host and microbe factors and may be triggered by strong or weak host responses to microbes (Casadevall and Pirofski 2003). Various microbially produced compounds have been shown to promote microbial fitness while either challenging (Fig. 2C) or supporting (Fig. 2D) host fitness. For example, Pseudomonas syringae can induce systemic susceptibility in host tissues by blocking host salicylic acid pathways, thereby enabling establishment and ultimately facilitating necrotrophic activity by the microbe (Newton et al. 2010). Likewise, sensitivity to microbe-associated molecules such as chitin can attenuate plant immune response and facilitate establishment by beneficial or antagonistic microbes. Symbiotic fungi, whether mutualistic or pathogenic, make use of effector proteins to attenuate plant immune response and facilitate establishment of symbiosis (Smith et al. 2017). In contrast, Epichlö spp. are fungal endophytes that produce alkaloids to deter herbivory and may activate plant systemic resistance, thereby improving plant host fitness (Saikkonen et al. 2016). By supporting plant growth and productivity, these endophytes are ensuring access to nutrient-rich habitats within plant tissues, supporting their own survival. Many studies have provided detailed, complex, and well-defined explanations of plant-microbe interactions. Despite these efforts, we are far from understanding how plants concomitantly manage pathogenic and beneficial interactions to ensure survival and maximize fitness. The eco-evolutionary perspective of the continuum-of-symbiosis framework is well suited for shaping research questions and objectives to advance our understanding of how plant-microbe cross-talk shapes symbiosis outcomes.

\section{ENVIRONMENTAL CONDITIONS SHAPE A CONTINUUM OF HOST-MICROBE INTERACTIONS}

The continuum-of-symbiosis approach recognizes that environmental factors contribute to patterns of differing ecologies between 
plants and microbes (Johnson et al. 1997, 2010; Klinger et al. 2016; McDonald et al. 2020; Rudgers et al. 2020; Saikkonen et al. 2020). Variation in nutrient accessibility and availability give rise to distinct microenvironments that guide microbial strategies for metabolism and growth and act as signaling cues for plants, animals, and microbes that give rise to differential life strategies (Fig. 2).
Dynamic and adaptable life strategies are important for enabling microbial fitness across a range of environmental conditions, with varying impacts on host fitness (Fig. 1B). For example, resource trade between plants and arbuscular mycorrhizal fungi (AMF) are important for maintaining symbiosis. Plant productivity coupled with soil phosphorus (P) supply will inform how beneficial this

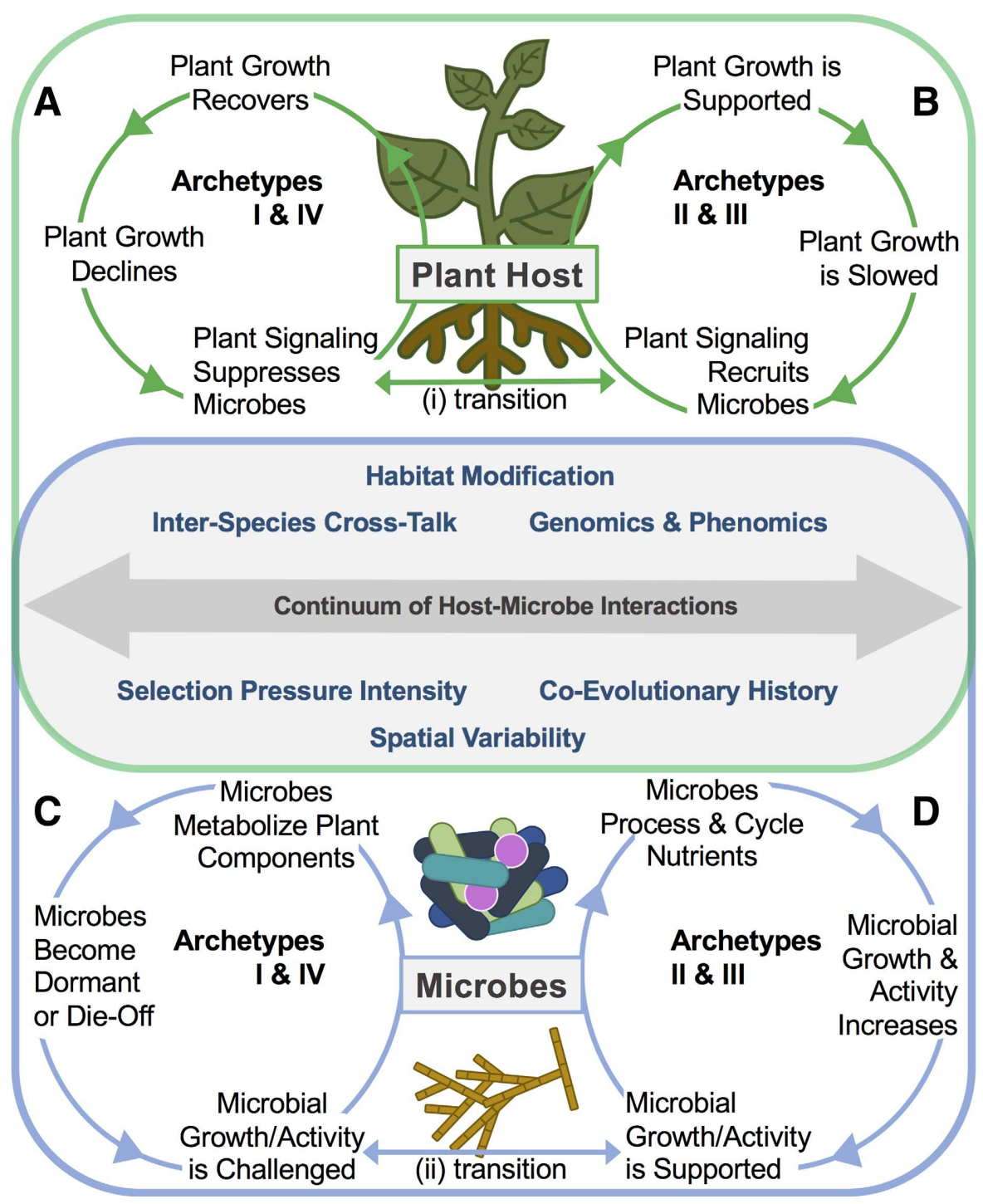

Fig. 2. Environment and ecology influence plant-microbe coevolution that drives diversity in symbiosis outcomes. Coevolutionary selection pressures include environmental constraints and ecological interactions that bring about changes in plant and microbe growth, activity, and gene expression to affect overall fitness and survival. Transient and stable plant-microbe associations arise from these gene by gene by environment $(G \times G \times E)$ interactions and can give rise to symbiosis outcomes that $\mathbf{A}$ and $\mathbf{C}$, are mutually exclusive or $\mathbf{B}$ and $\mathbf{D}$, support benefits to both host and microbes. Depending on the pressures and constraints, host organisms or microbes may transition between antagonistic (A and $C)$ and beneficial (B and D) associations, as indicated by the transition arrows ( $\mathrm{i}$ and ii). This continuum of host-microbe interactions is shaped by myriad factors, including the six outlined in the middle gray box. Examples of scenarios that may give rise to dominant archetypes of interactions include the following. A, Plant benefits at cost to microbe through either archetype I (exploitive acclimatization) as a highly plastic response to environmental pressures or host immune priming via induced systemic resistance or archetype IV (competitive adaptation) as a genetic or metabolic mechanism developed through coevolutionary competition such as systemic acquired resistance. B, Plant and microbe both benefit through either archetype II (complementary acclimatization) as microbes respond to plant phytohormone signaling to colonize the rhizosphere and exclude other potentially pathogenic microbes or archetype III (reciprocal adaptation), wherein coadaptation by the host plant and a specific microbe such as arbuscular mycorrhizal fungi facilitates nutrient exchange and acquisition. C, Microbe benefits at cost to plant either through archetype I (exploitive acclimatization), wherein the microbe expresses a specific suite of genes that attenuate host immune responses and enable metabolism of carbon or nitrogen through degradation of living plant tissues, or archetype IV (competitive adaptation), where specific microbes (such as Magnaporthe oryzae in rice) have evolved specialized genes or mechanisms to invade host tissue and acquire nutrients through parasitism. $\mathbf{D}$, Microbe and plant both benefit through either archetype II (complementary acclimatization), such that microbial mining of plant residues facilitates carbon and nitrogen cycling to enrich and stabilize the soil habitat for subsequent microbial and plant growth, or archetype III (reciprocal adaptation), whereby specific microbes are selected for via evolutionarily stabilized host-environment interactions such as nodule forming rhizobium in legume roots to support nitrogen fixation. 
exchange is with a symbiotic range that shifts among commensalism (archetype $\mathrm{V}$ ) when $\mathrm{P}$ is not limiting, to strong mutualisms (archetype III) when the host plant is productive despite P limitation, and possibly to parasitism (archetype II) under conditions of high plant carbon supply and nutrient-rich soil (Johnson et al. 2010) (Fig. 1B-f). Similarly, ectomycorrhizal fungi may switch between saprotrophy and biotrophy depending on resource availability (Smith et al. 2017). These dual metabolic capabilities enable a high degree of plasticity that contributes to a broader symbiotic range capable of supporting microbial growth under diverse environmental conditions (Fig. 1B-g).

Stress from drought, temperature, and salinity also shape plant-microbe interactions by altering microbial niches and triggering plant responses. Epichlö̈ endophytes may display more positive effects (archetype III) under nutrient-rich conditions, may exist between plant cells neutrally (archetype V), or may negatively affect plant production (archetype IV) in response to nutrient limited conditions (Saikkonen et al. 2016). Changes in phytohormone signaling such as increased carbohydrate production and abscisic acid synthesis in some roots can diminish host ability to resist antagonism (Jones et al. 2019). Additionally, the combined effect of environmental stress and altered host signaling can create shifts in overall community composition and elicit responses in specific taxonomic groups (Rudgers et al. 2020; Trivedi et al. 2020). For example, under drought conditions, Actinobacteria are commonly enriched and, under high salinity conditions, the fungal family Montagnulaceae is enriched in the rhizosphere (Jones et al. 2019). These observations are hypothesized to support nutrient acquisition through resource exchange (archetype II), arise from strategies for higher microbial persistence under stressors such as sporulation (archetype V), or may be the result of coevolutionary patterns that enable persistence of these microbes under adverse conditions (archetype III).

Microbial communities respond to fluctuating environments and may display features of resiliency-retaining both function and community composition - as well as historical contingency, where community membership and function are altered based on prior habitat conditions (Hawkes and Kiett 2015). Legacy effects from the soil environment and plant inputs can alter the regional species pool and give rise to distinct assemblages of microbial communities with varying degrees of adaptability and plasticity (Hawkes and Kiett 2015; Rudgers et al. 2020). These communities will further shape environmental conditions through niche modification and cycling of nutrients (Toju et al. 2018). In this way, resident microbial communities influence rhizosphere assembly and give rise to different patterns of succession across plant growth stages, which may alter the fate of plant-health outcomes. Wei et al. (2019) showed that the initial rhizosphere communities of healthy plants gave rise to more stable communities over plant growth stages, with higher network complexity and more diverse functional genes present than compared with the rhizospheres of diseased plants. Thus, microbe-environment interactions at the community level provide important context for evaluating host-microbe interactions spanning the continuum of symbiosis. This eco-evolutionary framework holds great promise for extrapolating meaningful conclusions about variability and stability in microbial communities across habitat conditions, including the potential functional consequences of certain host-microbe-environment associations.

\section{SPATIOTEMPORAL DYNAMICS INFLUENCE THE STABILITY OF HOST-MICROBE ASSOCIATIONS}

Host-microbe interactions vary over spatial and temporal scales, giving rise to context-dependency, spatial mismatch, and temporal mismatch that influence symbiosis outcomes (Rudgers et al. 2020). For example, a microbe acting as a commensal or mutualistic organism may shift to a pathogenic role to overcome immediate challenges to meet demands for growth and activity (Fig. 2C). These context-dependent associations may be transitory strategies (acclimatization) employed by microbial organisms (Newton et al. 2010) or occur over longer time periods (adaptation), giving rise to alternative stable states that drive patterns of plant-microbe symbioses (Douglas 2008). Using an experimental evolution approach, Morella et al. (2020) showed that fungal and bacterial communities in the tomato phyllosphere become less diverse and more stable over a few generations. This time-passage experiment demonstrates that temporal stability at a community level can arise over relatively short times scales (several generations as opposed to millions of years), highlighting the importance of temporal scales in shaping microbial adaptations to a host environment.

Additionally, there is strong evidence for the role of temporal scales in microbe-host mutualisms, whereby environmental conditions can favor mutualistic behavior, dissuade microbial cheaters, and reduce conflict or direct competition between microbes (Dethlefsen et al. 2007). Over longer time scales, symbiotic organisms may revert to free-living organisms as selection pressures change (Douglas 2008). Similarly, host-microbe mutualisms may break down over time due to changes in the distribution of plant species ranges (spatial mismatch) or decouple over short time scales following climate perturbations (temporal mismatch). Legume-rhizobium mutualisms are one example of a tightly linked symbiosis that may break down across spatial scales or over time (Douglas 2008). Under prolonged nitrogen-rich conditions, legume-rhizobium partnerships show lower beneficial qualities, possibly due to "cheater" strains gaining greater fitness benefits or due to a collapse in partner quality as rhizobia shift toward saprotrophy under nutrient-abundant environments (Klinger et al. 2016). Temporal variability acts as a significant driver of trade-offs in microbial lifestyle that can either support or break down stabilized associations. This may appear as the symbiotic range extending to encompass both reciprocal (archetype III) and competitive (archetype IV) coadaptation with plant hosts, or as a decoupling of coassociations toward commensalism (archetype V) and even transient acclimatization (archetype I, exploitive and archetype II, complementary).

Variability in symbiosis outcomes across spatial and temporal scales also shapes coevolution of hosts and microbes. These dynamics can support reciprocal adaptation (archetype III) such as legume-rhizobium mutualisms (Fig. 1B-e) (Klinger et al. 2016), or contribute to tightly linked host-specific competitive adaptation (archetype IV), as seen in Magnaporthe oryzae invasion of rice (Fig. 1B-h) (Quoc and Chau 2017). Several conceptual frameworks have emerged to improve study and characterization of host-microbe coevolution (McDonald et al. 2020), including the geographic mosaic of coevolution (Fernandes et al. 2019) and phylosymbiosis (Kohl 2020).

The geographic mosaic of coevolution explains that natural selection pressures vary by environment due to changes in gene expression (gene by environment interactions). As such, coevolution is a process influenced by genetic (e.g., gene flow, genetic drift, new mutations, and so on) and ecological (hot spots and cold spots of reciprocal selection) interactions. Geographic mosaics have enabled detection of phenotypic clusters that vary across spatial habitats and drive different patterns of coevolution over time (Fernandes et al. 2019). This is a particularly useful framework for evaluating the continuum of host-microbe symbiosis, because it acknowledges the dynamic nature of local interactions that may give rise to stabilized associations under certain eco-environmental conditions but not others. For example, ericoid mycorrhizal (ERM) fungal symbionts 
demonstrate dual saprotrophic and mutualistic associations that likely provide hosts with greater ecological flexibility, while facilitating microbial survival across a range of soil conditions (Martino et al. 2018). In Australia, spatial variability due to increased fire frequency is thought to have driven the coevolutionary association (archetype III) between ERM and sclerophyllous Ericaceae, enhancing host fitness and supporting microbial survival in soil habitats that are nonuniform, highly localized, and distinct (Martino et al. 2018). Similarly, Johnson et al. (2010) described the use of geographic mosaics for elucidating specialized, local adaptation to distinct soil environments by AMF symbionts. In their reciprocal inoculation experiment, they found that locally adapted AMF produced more symbiotic exchange structures (arbuscules) and more carbon-sequestration structures (extraradical hyphae), enhancing grass host fitness and microbial fitness, respectively (Johnson et al. 2010). The high degree of variability across spatial scales, even for tightly linked mutualisms such as AMF, indicates the need for an eco-evolutionary framework capable of capturing both plasticity and stability in patterns of host-microbe associations.

Phylosymbiosis describes eco-evolutionary patterns whereby the compositional similarity of host-associated microbial communities parallels the phylogeny of related host species (Kohl 2020; McDonald et al. 2020). As such, distinctions between host species are made possible because the general structure of microbial communities are stabilized and predictable across spatiotemporal scales. This is a particularly useful conceptual framework for contextualizing the eco-evolutionary processes that shape microbiome assembly (e.g., priority effects, selection, and filtering), diversity (e.g., ecological drift and codiversification), and potential function across a range of host-microbe-environment contexts. In particular, codiversification of host and microbial symbionts, also called cophylogeny, can help researchers elucidate the fitness gains arising from these host-microbe associations (Kohl 2020). Although not all species of Epichlö have codiverged with pooid grass hosts, there is evidence of cophylogeny (Saikkonen et al. 2016). Colonization by Epichlö̈ spp. confers a competitive advantage to host plants by boosting fitness. This benefit likely precipitated the spread of the microbial symbiont throughout the plant host range, enabling codiversification of plant and microbe species (Saikkonen et al. 2016). Despite this phylogenetic signal, there are transient changes that can significantly alter community composition and influence host performance. By using amplicon sequencing and meta-"omic" methods, researchers can capture "snapshots" of microbial interactions that are indicative of healthy or unhealthy states (Wei et al. 2019). Furthermore, whole-genome sequencing and comparative genome analysis has enhanced understanding of how cophylogeny shapes symbiosis outcomes (Saikkonen et al. 2016). Evaluating these environmental, ecological, and evolutionary characteristics through the continuum-of-symbiosis framework holds enormous potential for extrapolating meaningful conclusions across diverse study systems.

\section{GENETIC AND FUNCTIONAL TRAITS DIFFERENTIATE MICROBIAL LIFE HISTORY STRATEGIES}

The functional consequences of host-microbe associations reflect complex gene-gene-environment $(\mathrm{G} \times \mathrm{G} \times \mathrm{E})$ interactions (Fernandes et al. 2019). The influence of these interactions on symbiosis outcomes can be evidenced in microbial genomic capabilities and microbe-microbe communication. Specific genomic characteristics such as suites of genes have been shown to enable organisms to inhabit specific ecological niches (Karpinets et al. 2014; McDonald et al. 2020; Toju et al. 2018). Although the degree of redundancy in metabolic pathways can make distinctions based on gene expression and content difficult (Martino et al. 2018), microbial functional pathways may provide a means to characterize the ecological roles of certain groups. For example, bacterial genes capable of performing nitrogen fixation are more likely to be found in organisms living as mutualists than pathogens (Karpinets et al. 2014).

Among plant-associated bacteria, more than 1,000 biosynthetic gene clusters have been identified and include genes related to antibiotic production or antibiotic resistance, including terpenes, peptide synthetases, and polyketide synthases (Trivedi et al. 2020). Terpene biosynthesis is of particular interest to host-microbe fitness given its implication in interkingdom signaling, whereby strong responses by plant hosts facilitate chemical defense against pathogens and herbivores (Trivedi et al. 2020). Fungi exploit chemical defense signaling as a way to expand symbiotic ranges, which may provide advantages for host fitness in some cases. For example, the production of alkaloid compounds (e.g., lolines, peramine, indole-diterpenes, and ergot alkaloids) by cool-season grass hosts are mediated by the genomic content (intact genes, gene clusters, and gene fragments) and the arrangement of genes within a locus of the Epichlöe endophyte (Saikkonen et al. 2016). It is important to note that this tightly linked mutualism (archetype III) can be destabilized (archetype IV or V) because some Epichlö spp. are able to transition from asexual to sexual life cycles. Thus, the roles of host genetics, environmental conditions, and microbial life stages are important in shaping symbiosis outcomes that range from stabilized, coadaptive mutualism (archetype III) to evolutionary-linked conflict-induced antagonism (archetype IV), as well as commensalism (archetype V).

A key biological consideration for interpreting the 'omics of symbiosis is the understanding that microbial organisms demonstrate various life stages or life cycles. As such, there has been great interest in identifying the gene families and metabolic cascades involved in the transition from free living to plant associated (Dethlefsen et al. 2007; Newton et al. 2010; Plett and Martin 2011; Saikkonen et al. 2016; Trivedi et al. 2020). For example, many ECM fungi have the genomic capabilities to survive as free-living organisms in the soil by acting as weak saprotrophs but, when living symbiotically, show extensive deletion of plant cell-wall-degrading genes and upregulation of gene families useful for plant-microbe nutrient exchange (e.g., nutrient transporters, aquaporins, and amino acid permeases) (Plett and Martin 2011). Thus, there exist detectable genetic differences among plant-ECM pairs that can be used to discriminate mutualisms (archetype III) from commensalisms (archetype V) and antagonisms (archetype I).

Horizontal gene transfer is another important mechanism whereby microbes enhance their fitness under a range of ecoenvironmental conditions and contribute to different fitness outcomes in host organisms. Plasmids are particularly important in this process, enabling microbes to transition between lifestyles to maximize their fitness and, thus, expand their symbiotic range with varying impacts on host health (Fig. 1B). For example, acquisition of a plasmid containing virulence genes transformed Rhodococcus from a commensal (archetype V) or root-growth-promoting mutualist (archetype II) into a plant antagonist (archetype I) causing leafy galls in Nicotiana benthamiana (Savory et al. 2017). Virulence plasmids likely promote microbial fitness by enabling greater metabolic plasticity across a range of environmental conditions. Symbiotic plasmids facilitate similar adaptability among microbes and can stabilize coadaptive cooperation among plant-microbe mutualisms. For example, the pSym plasmid of rhizobia is important in maintaining microbial partner quality with legume hosts (Klinger et al. 2016). In high-nitrogen environments, decreased abundance for a suite of genes (nifH, nifA, nifD, fixC, and $\operatorname{nodB}$ ) in this plasmid give rise to less beneficial rhizobia (defective mutualists [archetype 
II] and not true cheaters [archetype I]). This demonstrates that environmental cues can affect microbial genetics to alter coevolutionary relationships (Fig. 1B-e).

Individuals in a community can simultaneously engage in cooperation and competition as part of reaching locally adaptive fitness optima (Dethlefsen et al. 2007). As such, there are selective pressures acting on microbial genetics, which can significantly shape interaction phenotypes with hosts. Microbial cross-feeding, and quorum sensing in particular, is an important feature of microbe-microbe signaling that contributes to different symbiosis outcomes (Newton et al. 2010; Trivedi et al. 2020). In general, Newton et al. (2010) noted that quorum sensing may be important for mediating lifestyle transitions, as has been demonstrated for $P$. syringae causing bacterial speckle of tomato (Fig. 1B-a). Latency enables Pseudomonas spp. to reside within host cells, living commensally (archetype V) until a time where the environment shifts and microbe-microbe communication triggers the switch to pathogenicity (archetype I). More recently, metagenomic analysis revealed an enrichment of homoserine lactone in plant-associated microbiomes, which is an important signaling molecule for quorum sensing (cooperation) and quorum quenching (interference). Quorum sensing among Enterobacter spp. can mediate the formation of physical barriers (root hair endophyte stack) in finger millet to exclude Fusarium graminearum (Trivedi et al. 2020). Thus, microbe-microbe communication in mixed communities has pronounced impacts on the symbiosis outcomes of a single microbe-host pair. Furthermore, bacterial communities associated with healthy tomato plants showed enrichment in antimicrobial genes (peptide and polyketide synthase genes) and demonstrated higher pathogen blocking (i.e., inhibited growth) of Ralstonia solanacearum (Wei et al. 2019).

Plant host features and spatiotemporal variability play an important role in altering microbial gene abundance and expression, which have functional consequences for symbiosis outcomes. This highlights a major challenge to characterizing host-microbe interactions: namely, that symbiosis outcomes arise from communities of microbes (rhizosphere, endosphere, and phyllosphere) interacting with a plant host that is part of a broader community, in a spatiotemporally variable landscape. These community-level ecological processes (e.g., competition, dispersal, and priority effects) influence microbial fitness and act as selection pressures, giving rise to distinct genomic features and functional traits. Thus, applying an eco-evolutionary framework such as the continuum-of-symbiosis approach described here is vital for teasing apart complexity to identify patterns and unifying processes that drive host-microbe symbiosis.

\section{WAYS FORWARD: APPLYING THE CONTINUUM TO RESEARCH AND TEACHING EFFORTS}

Relationships between a host organism and the members of its associated microbiome can be challenging to assess because they are among the most complex in studies of ecology and evolution (Dethlefsen et al. 2007; Kohl 2020; Saikkonen et al. 2016). Environmental signals, spatiotemporal dynamics, host health and genetics, microbial genomic content, and the myriad of cross-talk between microbes, plants, and the environment (Fig. 2) all contribute to the diversity of interactions that fall outside of singular or static categories (Fig. 1A). Microbes often do not solely rely on a single lifestyle strategy but, rather, can acclimatize to their environment and survive conditions not always suitable to their primary lifestyle (Martino et al. 2018; Saikkonen et al. 2020; Savory et al. 2017; Smith et al. 2017). Furthermore, depending on the host they associate with or the constraints of the environment, certain microbes can exhibit distinct and diverse lifestyle strategies that may be better described as symbiotic ranges (Fig. 1B). In light of this complexity, we suggest two approaches for improving understanding of the natural history of organisms and how these dynamics shape pathogenesis and mutualisms between microbes and hosts.

First, we envision a multitude of studies evaluating the breadth of microbe-host-environment relationships across temporal and spatial scales. Diversity surveys will provide key foundational knowledge of potential niches and symbiotic ranges that can inform subsequent hypothesis testing (Saikkonen et al. 2016; Toju et al. 2018). Furthermore, considering a changing climate, these studies will be important for determining the possibilities of spatial and temporal mismatch that may shape ecosystem resiliency (Hawkes and Kiett 2015; Rudgers et al. 2020; Saikkonen et al. 2020). Coupling compositional data with measures of potential function (e.g., metagenomics and metatranscriptomics) and plant-microbe signaling (e.g., secretome) will be useful for characterizing the functional attributes of plant-microbe symbiosis (Karpinets et al. 2014; Martino et al. 2018; Saikkonen et al. 2020; Wei et al. 2019). Finally, patterns of host-microbe coadaptation can be evaluated through experimental evolution studies that aim to understand changes in communities over time under controlled conditions (McDonald et al. 2020; Morella et al. 2020). These types of studies have already provided important insight into the stability of hostassociated microbiomes over generations, and the functional consequences of these microbial communities on host health (Morella et al. 2020).

Second, we suggest a combination of greenhouse and field studies for evaluating patterns of symbiosis and the influence of specific environmental and host cues on microbe-host fitness outcomes. Greenhouse studies are particularly well suited to investigate potential mechanisms that give rise to different structure-function relationships that may shape symbiosis outcomes. For example, 'omics data from controlled experiments provides insight into potential signaling molecules or microbial partnerships (e.g., keystone taxa) that shape microbe-microbe and microbe-plant cross-talk (Jones et al. 2019; Trivedi et al. 2020). Lab-based studies are well suited to explore dynamics of microbiome assembly and stability to tease apart how historical contingency and microbe-microbe interactions shape host health outcomes (Chaparro et al. 2014; Morella et al. 2020; Wei et al. 2019). These studies, however, are challenged by unrealistic containment of plant roots, especially beyond the seedling stage, that affect microbe-host interaction phenotypes. Thus, coupling targeted greenhouse studies with field studies is important for scaling research findings to real-world contexts and elucidating the range of variability in patterns of symbiosis (Savory et al. 2017). Furthermore, experimental field studies with welldocumented environmental characteristics will be crucial for assessing how complex ecosystem-level dynamics shape lifestyle changes in microbial inhabitants (Johnson et al. 2010; Klinger et al. 2016; Lofgren et al. 2018; Toju et al. 2018). These include evaluation of how plant diversity or plant species identity in cropping systems and environmental stressors such as drought or nutrient deficiency give rise to distinct phylogenomic partnerships (Saikkonen et al. 2016) and geographic mosaics of host-microbe coevolution (Fernandes et al. 2019; Johnson et al. 2010). Field studies will be particularly important for testing the efficacy (e.g., plant-growth-promoting microbes) and potential controls (e.g., phytohormone controls on rhizosphere function and diversity) arising from greenhouse findings. In an agricultural context, plant genotypes have been bred to maximize yield and minimize variability to improve agronomic efficiency. However, if we better understand the nature and control of lifestyle-change triggers between host and microorganism, 
it might be more effective and sustainable to develop breeding or crop-protection programs that aim to manipulate trigger signals to favor more symbiotic, mutualistic lifestyles (Newton et al. 2010; Saikkonen et al. 2016, 2020).

Although many researchers already recognize the importance of plasticity in host-microbe interactions, much of our discourse and materials for research, communication, and teaching overemphasize clearly delineated life strategies in specific microbe-host pairs (Box 1). Research outcomes will benefit greatly from designing experiments and contextualizing results within broader ecological and evolutionary patterns that better reflect the plasticity observed in plant-microbe interactions. Furthermore, reevaluating how we select examples and describe these complex host-microbe dynamics in our teaching and training efforts will be important for equipping students to better understand natural diversity and the eco-evolutionary processes that shape species interactions. Modifying the conceptual framework used to classify organisms will facilitate greater applicability of educational content to research and management efforts. To this end, a shift in language moving away from labeling an organism as strictly a pathogen or a mutualist and instead referring to microbes as "acting as a pathogen" or "acting as a mutualist" may be a more appropriate approach.

The continuum-of-symbiosis paradigm is essential for improving our prediction of plant-microbe symbiosis outcomes. We propose that rethinking symbiosis in terms of symbiotic ranges using clearly defined dominant archetypes will enhance research and teaching outcomes. By recognizing the roles of adaptability and stability among plant symbionts, research efforts can provide more holistic eco-evolutionary context to key plant-microbe relationships. Integrating these interdisciplinary perspectives will be essential for addressing core knowledge gaps in basic and applied research, including areas of disease pathogenesis, microbial ecology, and symbiosis.

\section{ACKNOWLEDGMENTS}

This commentary was partially drafted by participants in a journal club which was held during spring 2018. It was then revisited and reworked by A. Stengel during the viral pandemic of 2020. The schedule and repository of notes and readings from the journal club can be found here: https://github.com/ HerrLab/2018_Spring_Journal_Club. We thank the editors and all of the anonymous reviewers who provided input that improved this manuscript during the review process.

\section{LITERATURE CITED}

Brunner, F. S., Deere, J. A., Egas, M., Eizaguirre, C., and Raeymaekers, J. A. 2019. The diversity of eco-evolutionary dynamics: Comparing the feedbacks between ecology and evolution across scales. Funct. Ecol. 33:7-12.

Casadevall, A., and Pirofski, L. A. 2003. The damage-response framework of microbial pathogenesis. Nat. Rev. Microbiol. 1:17-24.

Chaparro, J. M., Badri, D. V., and Vivanco, J. M. 2014. Rhizosphere microbiome assemblage is affected by plant development. ISME J. 8: 790-803.

Dethlefsen, L., McFall-Ngai, M., and Relman, D. A. 2007. An ecological and evolutionary perspective on human-microbe mutualism and disease. Nature 449:811-818.

Douglas, A. E. 2008. Conflict, cheats and the persistence of symbioses. New Phytol. 177:849-858.

Fernandes, L. D., Lemos-Costa, P., Guimarães, P. R., Thompson, J. N., and de Aguiar, M. A. M. 2019. Coevolution creates complex mosaics across large landscapes. Am. Nat. 194:217-229.

Hawkes, C. V., and Kiett, T. H. 2015. Resilience vs. historical contingency in microbial responses to environmental change. Ecol. Lett. 18:612-625.
Johnson, N. C., Graham, J. H., and Smith, F. A. 1997. Functioning of mycorrhizal associations along the mutualism-parasitism continuum. New Phytol. 135:575-585.

Johnson, N. C., Wilson, G. W. T., Bowker, M. A., Wilson, J. A., and Miller, M. 2010. Resource limitation is a driver of local adaptation in mycorrhizal symbioses. Proc. Natl. Acad. Sci. U.S.A. 107:2093-2098.

Jones, P., Garcia, B. J., Furches, A., Tuskan, G. A., and Jacobson, D. 2019. Plant host-associated mechanisms for microbial selection. Front. Plant Sci. 10:862.

Karpinets, T. V., Park, B. H., Syed, M. H., Klotz, M. G., and Uberbacher, E. C. 2014. Metabolic environments and genomic features associated with pathogenic and mutualistic interactions between bacteria and plants. Mol. Plant-Microbe Interact. 27:664-677.

Klinger, C. R., Lau, J. A., and Heath, K. D. 2016. Ecological genomics of mutualism decline in nitrogen-fixing bacteria. Proc. R. Soc. B 283: 20152563.

Kohl, K. D. 2020. Ecological and evolutionary mechanisms underlying patterns of phylosymbiosis in host-associated microbial communities. Philos. Trans. R. Soc. B 375:20190251.

Lofgren, L. A., LeBlanc, N. R., Certano, A. K., Nachtigall, J., LaBine, K. M., Riddle, J., Broz, K., Dong, Y., Bethan, B., Kafer, C. W., and Kistler, H. C. 2018. Fusarium graminearum: Pathogen or endophyte of North American grasses? New Phytol. 217:1203-1212.

Martino, E., Morin, E., Grelet, G. A., Kuo, A., Kohler, A., Daghino, S., Barry, K. W., Cichocki, N., Clum, A., Dockter, R. B., Hainaut, M., Kuo, R. C., LaButti, K., Lindahl, B. D., Lindquist, E. A., Lipzen, A., Khouja, H. R., Magnuson, J., Murat, C., Ohm, R. A., Singer, S. W., Spatafora, J. W., Wang, M., Veneault-Fourrey, C., Henrissat, B., Grigoriev, I., Martin, F. M., and Perotto, S. 2018. Comparative genomics and transcriptomics depict ericoid mycorrhizal fungi as versatile saprotrophs and plant mutualists. New Phytol. 217:1213-1229.

McDonald, J. E., Marchesi, J. R., and Koskella, B. 2020. Application of ecological and evolutionary theory to microbiome community dynamics across systems. Proc. R. Soc. B 287:20202886.

Morella, N. M., Weng, F. C. H., Joubert, P. M., Metcalf, C. J. E., Lindow, S., and Koskella, B. 2020. Successive passaging of a plant-associated microbiome reveals robust habitat and host genotype-dependent selection. Proc. Natl. Acad. Sci. U.S.A. 117:1148-1159.

Newton, A. C., Fitt, B. D. L., Atkins, S. D., Walters, D. R., and Daniell, T. J. 2010. Pathogenesis, parasitism and mutualism in the trophic space of microbe-plant interactions. Trends Microbiol. 18:365-373.

Plett, J. M., and Martin, F. 2011. Blurred boundaries: Lifestyle lessons from ectomycorrhizal fungal genomes. Trends Genet. 27:14-22.

Quoc, N. B., and Chau, N. N. B. 2017. The role of cell wall degrading enzymes in pathogenesis of Magnaporthe oryzae. Curr. Protein Pept. Sci. $18: 1019-1034$

Rudgers, J. A., Afkhami, M. E., Bell-Dereske, L., Chung, Y. A., Crawford, K. M., Kivlin, S. N., Mann, M. A., and Nuñez, M. A. 2020. Climate disruption of plant-microbe interactions. Annu. Rev. Ecol. Evol. Syst. 51: 561-586.

Saikkonen, K., Nissinen, R., and Helander, M. 2020. Toward comprehensive plant microbiome research. Front. Ecol. Evol. 8:61.

Saikkonen, K., Young, C. A., Helander, M., and Schardl, C. L. 2016. Endophytic Epichlö species and their grass hosts: From evolution to applications. Plant Mol. Biol. 90:665-675.

Savory, E. A., Fuller, S. L., Weisberg, A. J., Thomas, W. J., Gordon, M. I., Stevens, D. M., Creason, A. L., Belcher, M. S., Serdani, M., Wiseman, M. S., Grünwald, N. J., Putnam, M. L., and Chang, J. H. 2017. Evolutionary transitions between beneficial and phytopathogenic Rhodococcus challenge disease management. eLife 6:e30925.

Smith, G. R., Finlay, R. D., Stenlid, J., Vasaitis, R., and Menkis, A. 2017. Growing evidence for facultative biotrophy in saprotrophic fungi: Data from microcosm tests with 201 species of wood-decay basidiomycetes. New Phytol. 215:747-755.

Toju, H., Peay, K. G., Yamamichi, M., Narisawa, K., Hiruma, K., Naito, K., Fukuda, S., Ushio, M., Nakaoka, S., Onoda, Y., Yoshida, K., Schlaeppi, K., Bai, Y., Sugiura, R., Ichihashi, Y., Minamisawa, K., and Kiers, T. 2018. Core microbiomes for sustainable agroecosystems. Nat. Plants 4:247-257.

Trivedi, P., Leach, J. E., Tringe, S. G., Sa, T., and Singh, B. K. 2020. Plant-microbiome interactions: From community assembly to plant health. Nat. Rev. Microbiol. 18:607-621.

Wei, Z., Gu, Y., Friman, V.-P., Kowalchuk, G. A., Xu, Y., Shen, Q., and Jousset, A. 2019. Initial soil microbiome composition and functioning predetermine future plant health. Sci. Adv. 5:eaaw0759. 